\title{
The Attenuation of Hard $\gamma$-rays in Strongly-Magnetized Pulsars
}

\author{
Alice K. Harding \\ Code 661, NASA/Goddard Space Flight Center, Greenbelt, MD 20771 \\ Matthew G. Baring \\ Code 661, NASA/Goddard Space Flight Center, Greenbelt, MD 20771 \\ Compton Fellow, Universities Space Research Association
}

\begin{abstract}
We investigate the spectral attenuation due to one-photon pair production and photon splitting in neutron star magnetospheres. The calculations are performed in a Schwarzschild metric, thereby including redshift and field enhancement effects associated with curved spacetime. We find that pair production and splitting totally inhibit emission above around $10-30 \mathrm{MeV}$ in PSR1509-58, whose surface field is inferred to be as high as $3 \times 10^{13}$ Gauss. Model pulsar spectra of splitting cascades for high field cases, where splitting is important, are consistent with the very low cutoff energy in PSR1509-58 for a wide range of polar cap sizes.
\end{abstract}

The spectra of $\gamma$-ray pulsars generally are observed to cutoff above a few $\mathrm{GeV}$ (e.g. Thompson, this volume). PSR1509-58 stands out as having both an unusually low spectral cutoff energy and the highest inferred surface magnetic field $\left(3 \times 10^{13}\right.$ Gauss). It has been detected by only the CGRO instruments (see Fig. 1 caption for references) operating in the lowest energy bands, BATSE and OSSE, with the higher energy instruments, COMPTEL and EGRET, giving upper limits that require a cutoff or turnover between 2 and $30 \mathrm{MeV}$. A natural question then arises: does the high field of this pulsar cause its spectrum to differ so much from that of the other $\gamma$-ray pulsars?

Gamma-rays from pulsars can be efficiently attenuated in their magnetospheres via the mechanism of single-photon pair production and also the exotic QED process of photon splitting, which become prolific in fields approaching the quantum critical value of $4.41 \times 10^{13}$ Gauss. Photon splitting $\gamma \rightarrow \gamma \gamma$, will operate in the high field regions near pulsar polar caps but has not yet been included in polar cap model calculations (see Harding, this volume). The rate of photon splitting increases rapidly with increasing field strength (Adler 1971), so that it may even be the dominant attenuation process in the highest field pulsars. Since photon splitting has no threshold, it can attenuate photons below the threshold for pair production, $\varepsilon=2 / \sin \theta_{k B}$, and can thus produce cutoffs in the spectrum at lower energies. Here $\theta_{k B}$ is the angle between the photon momentum and the magnetic field vectors, and $\varepsilon$ expressed in units of $m c^{2}$.

We have computed the expected shape and location of the cutoffs due to combined photon splitting and pair production in the $\gamma$-ray spectrum of PSR1509-58 by a Monte Carlo simulation of a photon splitting cascade (Hard- 


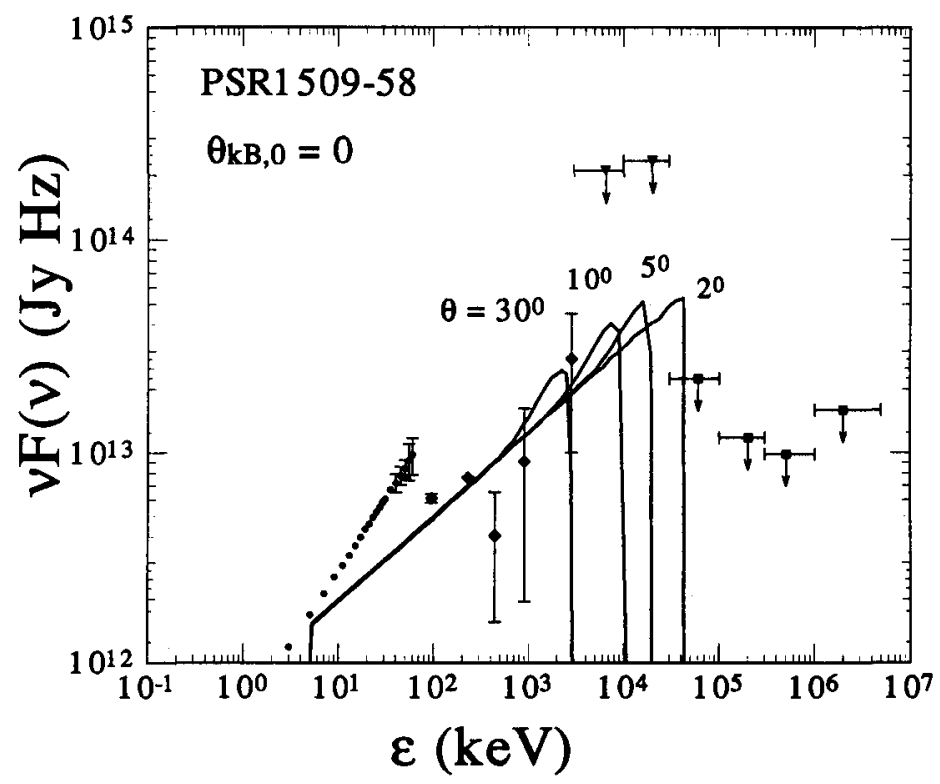

Figure 1. Polarization-averaged, photon splitting cascade spectra compared to the PSR1509-58 observed spectrum. Data points are from GINGA (Kawai et al. 1993): circles, OSSE (Matz et al. 1994): diamonds, COMPTEL (Carramiñana et al. 1995): triangles, and EGRET (Nel et al. 1996): squares.

ing et al. 1996). The spectra of such a cascade, for a photon input spectrum ( $\alpha$ $=1.6$ ) injected from the stellar surface parallel to the field at various magnetic colatitudes $\theta$, is shown in Figure 1. In these cascades, only the $\perp \rightarrow\|\|$ splitting mode is assumed to operate, so that $\perp$ mode photons may either pair produce or split into $\|$ mode photons. The $\|$ model photons may only pair produce. The polarized spectra show that cutoffs occur at slightly different energies for the two modes. Radiation from the pairs has not been included in these calculations. In order to produce a cutoff between 4 and $30 \mathrm{MeV}$, we find that $2^{0} \lessgtr \theta \leqslant 25^{0}$. These limits are consistent with the standard polar cap half-angle of $2.14^{0}$ for PSR1059-58.

\section{References}

Adler, S.L. 1971, Ann. Phys., 67, 599.

Carramiñana, A. et al. 1995, A \& A, in press.

Harding, A. K., Baring, M. G. \& Gonthier, P. L. 1996, ApJ, submitted.

Kawai, N. et al. 1993, AIP Conf. Proc. 280, 213.

Matz, S. M. et al.: 1994, ApJ, 434, 288.

Nel, H. I. et al. 1996, ApJ, in press. 